\title{
miR-30a Suppresses Cell Migration and Invasion Through Downregulation of PIK3CD in Colorectal Carcinoma
}

\author{
Ming Zhong Zhengqian Bian Zhiyong Wu \\ Department of General Surgery, Renji Hospital, Shanghai Jiaotong University School of Medicine, \\ Shanghai
}

\author{
Key Words \\ miR-30a • Colorectal carcinoma • Migration • Invasion $•$ PIK3CD
}

\begin{abstract}
Background/Aims: MicroRNAs (miRNAs) play key roles in tumor metastasis. The aim of this study was to determine the regulation and function of miR-30a in colorectal carcinoma (CRC) metastasis. Methods: The expression of miR-30a was detected in CRC cell lines and samples by qRT-PCR. The anti-metastatic effect of miR-30a was determined by both in vitro and in vivo assays. A luciferase reporter assay was performed to determine target association between miR-30a and phosphoinositide 3-kinase catalytic subunit delta (PIK3CD). Results: miR-30a was significantly downregulated in highly metastatic CRC cell lines and metastatic tissues. Overexpression of miR-30a suppressed CRC cell migration and invasion in vitro and liver metastasis in vivo, whereas miR-30a deletion dramatically promoted cell migration and invasion. Further studies revealed that PIK3CD is a direct target of miR-30a as miR-30a bounds directly to the $3^{\prime}$-UTR of PIK3CD, subsequently reducing its expression. Similar to the restoring miR-30a expression, PIK3CD downregulation inhibited cell migration and invasion, whereas PIK3CD overexpression rescued the suppressive effect of miR-30a. Moreover, significant downregulation of miR-30a in metastatic CRC tissues was found to be inversely correlated with PIK3CD expression. Mechanistic studies revealed that miR-30a down-regulated the expression of key components of the Akt/mTOR pathway, whereas PIK3CD overexpression reversed this negative effect. Conclusion: Our findings indicate that miR-30a might function as a metastasis suppressor in CRC. miR-30a may be a potential therapeutic target to block CRC metastasis.
\end{abstract}


Zhong/Bian/Wu: miR-30a Targets PIK3CD Expression in CRC

\section{Introduction}

Colorectal carcinoma (CRC) is the third leading cause of cancer-related deaths worldwide [1]. Despite achievements in the treatment in the few past decades, CRC remains a major public health concern, resulting in more than 600,000 deaths each year. The major cause of death and relapse from CRC is metastasis, however, promising therapy for CRC metastasis is not available. Given this, understanding the molecular mechanisms that promote metastasis in CRC is of crucial significance to the development of therapeutic strategies for CRC patients.

MicroRNAs (miRNAs) are a recently discovered class of small noncoding RNA molecules that modulate gene expression [2]. Accumulating evidence indicates that aberrant expression of miRNAs correlates with various human cancers, and that miRNAs can act as tumor suppressors or oncogenes [3,4]. Several studies have also reported that miRNAs are implicated in both the suppression and promotion of tumor metastasis [5]. For example, miR-143 inhibits cell invasion and metastasis in osteosarcoma, colorectal cancer and pancreatic cancer [6-8]; miR-139 targets IGF1R and inhibits in vitro and in vivo invasion and metastasis in colorectal cancer [9]; and miR-25 promotes cell migration and invasion in esophageal squamous cell carcinoma [10]. Recent studies showed that miR-30a is significantly downregulated in colorectal cancers [11-13]. The involvement of miR-30a in the metastasis of breast cancer and non-small cell lung cancer has been reported $[14,15]$; however, its roles and mechanism in CRC metastasis remain largely unknown.

In this study, we revealed significant downregulation of miR-30a in metastatic CRC cell lines and tissues. Overexpression of miR-30a suppressed CRC cell metastasis and invasion in vitro and in vivo, whereas miR-30a knockdown promoted CRC cell migration and invasion. Furthermore, we identified PIK3CD as a direct and functional target of miR-30a. We also confirmed an inverse correlation between miR-30a and PIK3CD expression in human CRC tissues. Our study reveals a crucial role of miR-30a-dependent PIK3CD modulation in CRC metastasis.

\section{Materials and Methods}

Cell culture and tumor specimens

The human colorectal cancer (CRC) cell lines, including SW480, CaCo-2, WiDr, HT29, LoVo, HCT116 and SW620, were purchased from American Type Culture Collection. Cells were cultured in RPMI1640 medium (Thermo Scientific HyClone, Beijing, China) supplemented with 10\% fetal bovine serum, 100 units $/ \mathrm{ml}$ of penicillin and $100 \mu \mathrm{g} / \mathrm{ml}$ of streptomycin. The cells were incubated in a $5 \% \mathrm{CO}_{2}$ atmosphere at $37^{\circ} \mathrm{C}$. Primary CRC samples and lymph node metastatic tissues were obtained from Renji Hospital, Shanghai Jiaotong University School of Medicine. Studies involving these tissues were approved by the Ethics Review Committee of the Institutional Review Board of the hospital, and written informed consent was obtained from every patient.

\section{RNA extraction and quantitative real-time PCR ( $q R T-P C R)$}

Total RNA was extracted using the TRIzol reagent (Invitrogen, Carlsbad, CA, USA) according to the manufacturer's protocol. cDNA was synthesized from $1 \mu \mathrm{g}$ of total RNA with a reverse reaction kit (Promega, Madison, WI, USA). The expression of PIK3CD and $\beta$-actin was quantified by qRT-PCR using SYBR-Green assays (TOYOBO, Tokyo, Japan) as previously reported [16]. To quantify the expression of mature miR30a, RT-PCR was performed using TaqMan microRNA assays (Applied Biosystems, Foster City, CA, USA) as described previously [17]. U6 snRNA was used as an endogenous control for miRNA detection. The relative expression levels of each gene were calculated and normalized using the $2^{-\Delta \Delta \mathrm{Ct}}$ method relative to $\beta$-actin or U6 snRNA. 
Zhong/Bian/Wu: miR-30a Targets PIK3CD Expression in CRC

TA-3' (reverse). PIK3CD siRNA was purchased from GeneChem (Shanghai, China). These sequences were separately cloned into the lentivirus-based expression plasmid pLenti6.3 (Invitrogen). Virus packaging and infection were performed according to standard protocols as recommended by the manufacturer. SW620 cells $\left(1 \times 10^{5}\right)$ were infected with $1 \times 10^{7}$ lentivirus transducing units in the presence of $10 \mu \mathrm{g} / \mathrm{ml}$ polybrene (Sigma, St Louis, Missouri, USA). Empty lentiviral vector was used as negative control. The inhibitors antimiR-30a and anti-control were purchased from Ambion (Austin, TX, USA). SW480 cells were transfected with anti-miR-30a and negative control using Lipofectamine 2000 (Invitrogen) according to the manufacturer's protocol. Cells were collected $48 \mathrm{~h}$ after transfection.

\section{Plasmid construction}

The coding sequences of PIK3CD were cloned into pcDNA3.1 (+) to generate PIK3CD expression vectors. The wild-type PIK3CD 3'UTR was cloned into the pMIR-REPORT luciferase vector (Ambion, Austin, TX, USA). Mutant PIK3CD 3'UTR was generated based on the pMIR-PIK3CD-3'UTR by mutating 3 nt that are recognized by miR-30a. The primers for PIK3CD were: $5^{\prime}$-TGT GGA TCC CTG TCA TCT GGG AAG TAA CAA CGC A-3' (forward) and 5'-AAG TCT AG AGA GCC ACT ACT GCC TGT TGT CTT TG-3' (reverse). The primers for PIK3CD 3'UTR were: 5' - T CTG AGC TCG CTA AAC AGC CAT AAA CGG AAA CGC-3' (forward) and 5'-ACC ACG CGT GCG TA GAT TCT CCT TTA TGG GGC TG-3' (reverse).

\section{Luciferase reporter assays}

The reporter plasmid was transiently transfected into SW620 and SW480 cells in the presence of either miR-30a or miR-control. After $48 \mathrm{~h}$, the cells were harvested and lysed, and luciferase activity was measured using the Dual-Luciferase Reporter Assay System (Promega, Madison, WI, USA). Renilla-luciferase was used for normalization. The experiments were performed independently in triplicate.

\section{Cell migration and invasion assays}

A 24-well transwell plate (Corning, New York, USA) was used to measure each cell line's migratory and invasive ability. For transwell migration assays, $5 \times 10^{4}$ cells suspended in serum-free medium were plated in the upper chamber lined with a non-coated membrane. For invasion assays, chamber inserts were coated with $150 \mu \mathrm{g}$ of Matrigel (BD Biosciences, Bedford, MD, USA). Then, $5 \times 10^{4}$ cells were plated in the upper chamber. The chambers were then inserted into the wells of a 24 -well plate and incubated at $37^{\circ} \mathrm{C}$ for $24 \mathrm{~h}$ in RPMI-1640 medium with $10 \%$ fetal bovine serum before examination. The cells remaining on the upper surface of the membranes were removed, whereas the cells adhering to the lower surface were fixed, stained in a dye solution containing $0.1 \%$ crystal violet, air-dried and counted under a microscope (Olympus Corp., Tokyo, Japan) to calculate their relative numbers. Results are presented as an average of triplicate experiments.

\section{Western blot analysis}

Cells were lysed with RIPA lysis buffer. Proteins were separated by sodium dodecyl sulfatepolyacrylamide gel electrophoresis, and transferred to polyvinylidene difluoride membranes (Millipore, Bedford, MA, USA). Blots were blocked and then probed with antibodies against PIK3CD (1:500 dilution; Santa Cruz Biotechnology, Santa Cruz, CA, USA), phospho-Akt (Ser473; 1:400 dilution; Santa Cruz Biotechnology), Akt (1:1000 dilution; Santa Cruz Biotechnology), phospho-mTOR (1:500 dilution; Cell Signaling Technology Inc., Beverly, MA, USA), mTOR (1:1000 dilution; Santa Cruz Biotechnology), $\beta$-actin (1:500 dilution; Cell Signaling Technology Inc.). After washing, the blots were incubated with horseradish peroxidase-conjugated secondary antibodies and visualized using super enhanced chemiluminescence detection reagent (Amersham Biosciences, Piscataway, NJ, USA).

\section{Animal studies}

Athymic BALB/c nude mice (4-6 weeks old, 18-22 g body weight) were randomly assigned to two groups (SW620-miR-30a or SW620-miR-control, 6 mice per group), and $1 \times 10^{6}$ cells were injected intravenously via tail vein. All mice were euthanized at 6 weeks after the initial injection, and the livers were excised to count the tumor nodules under a stereomicroscope (Olympus). The nude mice were provided by the Experimental Animal Center of Renji Hospital, Shanghai Jiaotong University School of Medicine. 


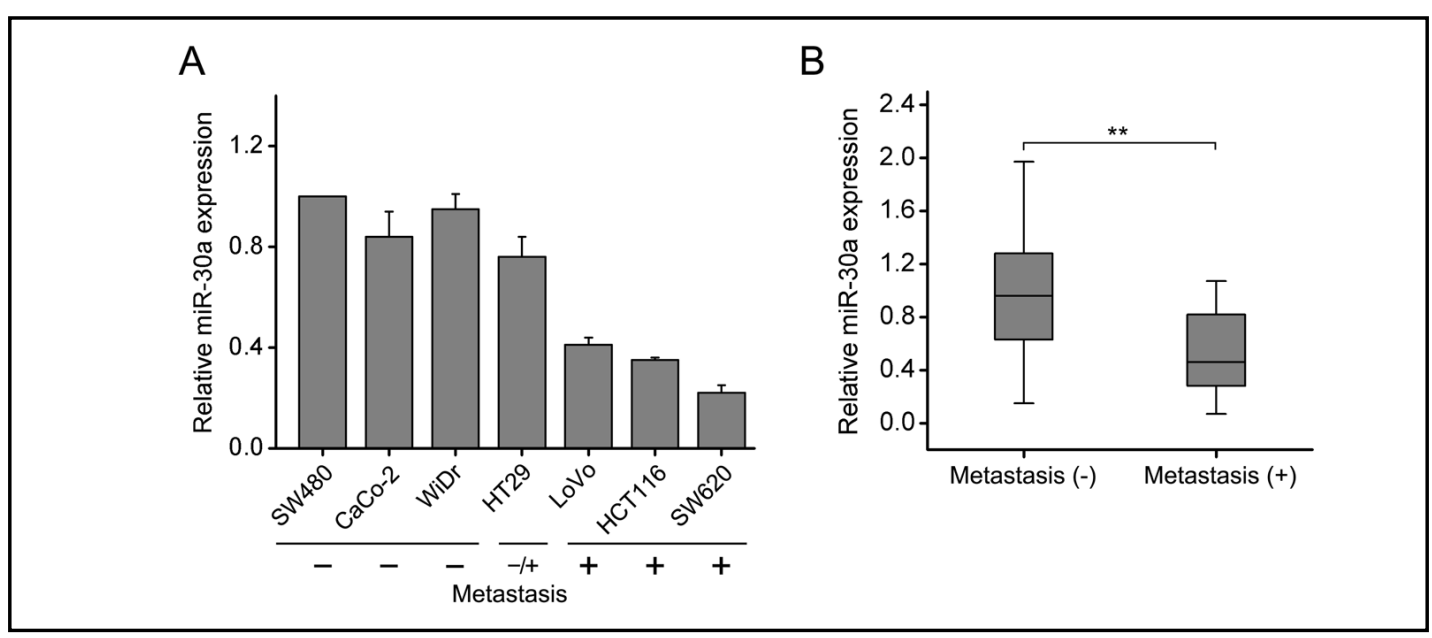

Fig. 1. Downregulation of miR-30a in metastatic CRC cells and lymph node metastatic tissues. (A) qRTPCR analysis of miR-30a expression in CRC cell lines with differential metastasis potential. (B) miR-30a expression in CRC with or without lymph node metastasis.

All animal studies complied with Shanghai Jiaotong University animal use guidelines and the protocols approved by Shanghai Jiaotong University Animal Care Committee.

\section{Statistical analysis}

Statistical analysis was performed using the SPSS13.0 program. Data were expressed as the means \pm SEM of at least three independent experiments. The Student's t-test was used to analyze differences between two groups. The relationship between miR-30a and PIK3CD mRNA expression was explored by Spearman's correlation. Differences were considered statistically significant at $\mathrm{p}<0.05,{ }^{*} \mathrm{p}<0.05$ and $* *$ $\mathrm{p}<0.01$.

\section{Results}

miR-30a is downregulated in metastatic CRC cells and lymph node metastatic tissues

To gain an insight into the roles of miR-30a in CRC metastasis, the expression levels of miR-30a were first examined in a panel of CRC cell lines showing differential metastasis potential by qRT-PCR. The results showed that inverse correlations between the levels of miR$30 \mathrm{a}$ and the metastasis potential of these cells (Fig. 1A). Furthermore, tissues from lymph node metastases showed lower levels of miR-30a than primary CRC tissues, indicating a potential metastasis-suppressive function of miR-30a in CRC (Fig. 1B). Taken together, these results suggest that downregulation of miR-30a is correlated with increased CRC metastasis.

Overexpression of miR-30a suppresses cell migration and invasion in CRC cells

To determine whether miR-30a can affect CRC cell migration and invasion, we stably overexpressed miR-30a in SW620 cells. These cells (with and without miR-30a overexpression) were then used to determine their migratory and invasive ability. As shown in Fig. 2A, overexpression of miR-30a significantly reduced the migration of SW620 cells in Transwell assays without Matrigel and suppressed the invasion of SW620 cells in Transwell assays with Matrigel. In contrast, the migration and invasion of SW480 cells were promoted when endogenous miR-30a was knocked down with antisense oligonucleotides (Fig. 2B). These results demonstrate that miR-30a can suppress CRC cell migration and invasion in vitro. To further investigate whether overexpression of miR-30a would act the same in vivo, miR-30a-overexpressed and scramble control SW620 cells were injected into nude mice 
A

A miR-control
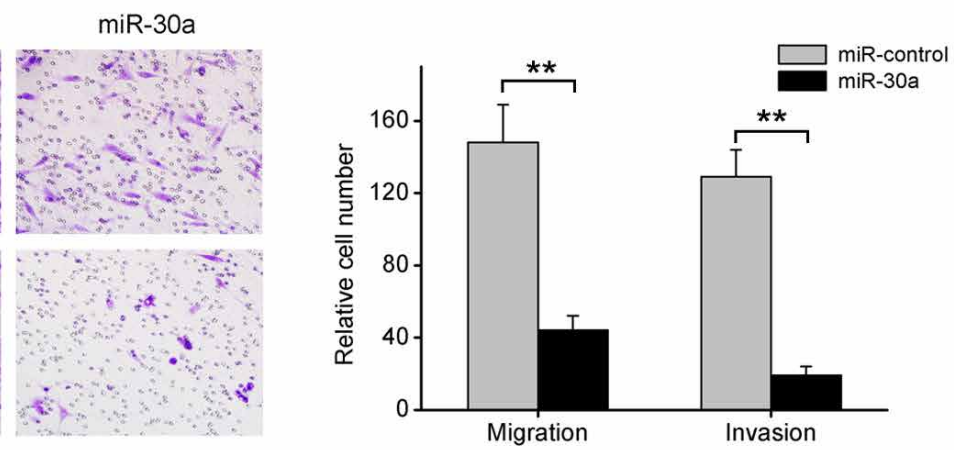

B

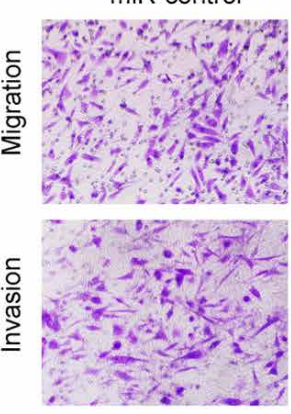

anti-miR-30a
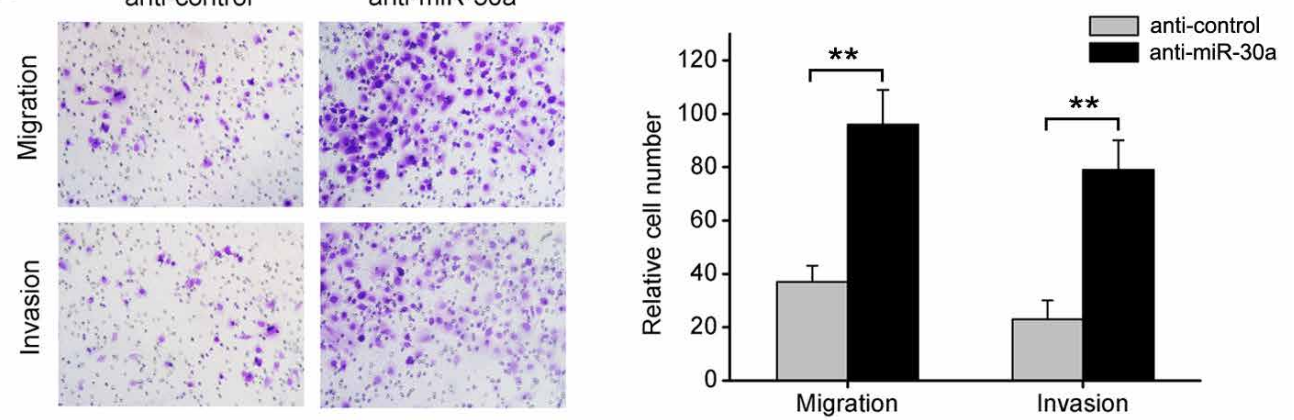

C

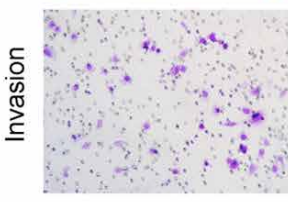

twot

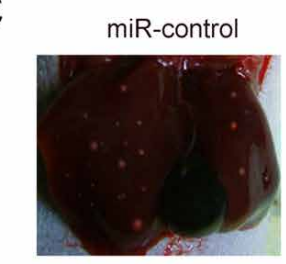

miR-30a
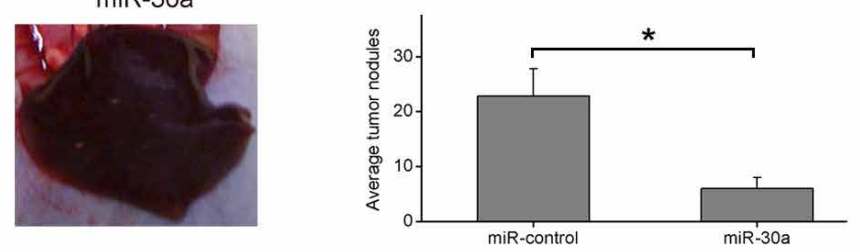

Fig. 2. miR-30a suppresses CRC cell invasion and metastasis in vitro and in vivo. (A) Overexpression of miR-30a significantly impeded cell migration and invasion in SW620 cells, in comparison with scramble controls. (B) Inhibition of miR-30a expression by transfecting miR-30a inhibitors promoted cell migration and invasion of SW480 cells, compared with scramble controls. (C) miR-30a affects liver metastasis as revealed by the experimental metastasis animal model. Representative images of livers from mice injected with SW620-miR-30a or SW620-miR-control are shown on the left. The number of macroscopic nodules were counted and analysed.

by intravenous tail injections. We found that the mean number of metastatic nodules on the surface of the liver was significantly repressed in SW620-miR-30a groups compared to negative controls (Fig. 2C). Taken together, these results indicate that stable overexpression of miR-30a is able to suppress CRC invasion both in vitro and in vivo.

\section{PIK3CD is a direct target of miR-30a}

To explore the mechanism by which miR-30a inhibits CRC metastasis and invasion, we next aimed to investigate the potential gene targets of miR-30a using target prediction programs, PicTar and TargetScan. Our analysis revealed that PIK3CD was a potential target of miR-30a. The 3'-UTR of PIK3CD mRNA contains a complementary site for the seed region of miR-30a (Fig. 3A). To verify whether or not PIK3CD is a direct target of miR-30a, a human 
A

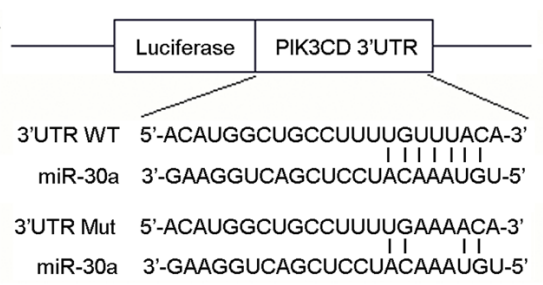

B

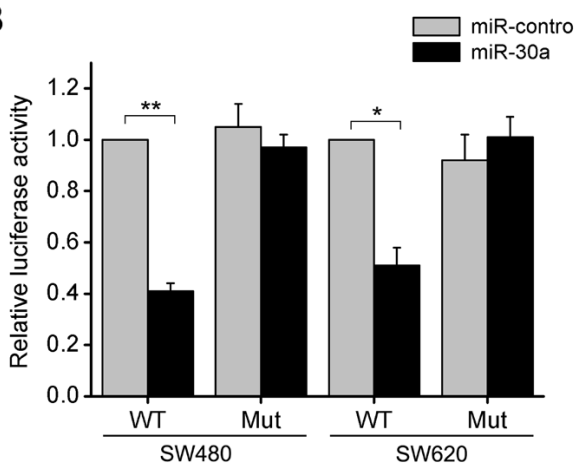

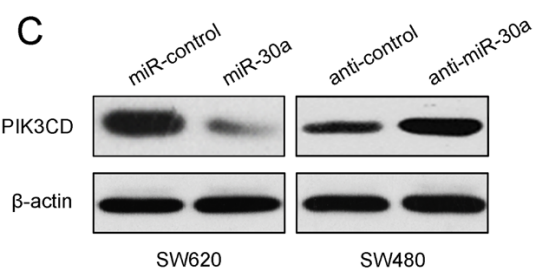

D

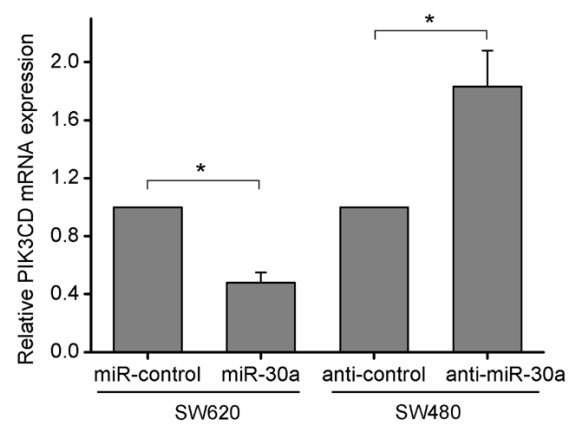

Fig. 3. PIK3CD is a direct target of miR-30a. (A) Putative miR-30a-binding site in the PIK3CD 3'UTR. A mutation was introduced into the PIK3CD 3'UTR sequence by altering $3 \mathrm{nt}$ that are recognized by miR-30a. Wild-type (WT) or mutant PIK3CD 3'UTR (Mut) was subcloned into a dual-luciferase reporter vector. (B) SW620 and SW480 cells were transfected with the luciferase reporter vector containing WT PIK3CD 3'-UTR or Mut PIK3CD 3'-UTR and infected by miR-control or miR-30a. Firefly luciferase activity was determined $48 \mathrm{~h}$ posttransfection and normalized to Renilla luciferase activity. (C) PIK3CD protein expression levels in CRC cells infected with miR-control or miR-30a were determined by Western blotting. (D) PIK3CD mRNA expression levels in CRC cells infected with miR-control or miR-30a were determined by qRT-PCR.

PIK3CD 3'UTR fragment containing the wild-type or mutant miR-30a-binding site was inserted downstream of the luciferase open reading frame. These reporter constructs were used to cotransfect SW480 and SW620 cells, which were infected by miR-control-lentivirus or miR-30a-lentivirus. As shown in Fig. 3B, the relative luciferase activity of the reporter containing wild-type PIK3CD 3'-UTR was markedly decreased upon miR-30a cotransfection, whereas the luciferase activity of the reporter containing the mutant binding site was unaffected (Fig. 3B). Furthermore, miR-30a overexpression decreased the levels of PIK3CD mRNA and protein, as determined by qRT-PCR and Western blotting (Figs. 3C and D). These results strongly suggest that PIK3CD is a direct target of miR-30a in CRC cells.

\section{PIK3CD is involved in miR-30a-inhibited CRC cell migration and invasion}

To further determine whether miR-30a regulated CRC cell migration and invasion through PIK3CD, we first transfected SW620 cells with siPIK3CD or the negative control. As shown in Fig. 4A, PIK3CD knockdown significantly inhibited SW620 cell migration and invasion. In contrast, PIK3CD overexpression in SW480 cells promoted cell migration and invasion (Fig. 4B). Subsequently, the effect of PIK3CD (without 3'-UTR) were examined in the miR-30a-overexpressed SW480 cells. The Transwell assays indicated that ectopically expressing PIK3CD significantly promoted the migration and invasion of miR-30aoverexpressed SW480 cells (Fig. 4C). To further verify whether miR-30a-induced modulation of PIK3CD is of clinical relevance, we analyzed the correlation between miR-30a and PIK3CD expression in $18 \mathrm{CRC}$ samples with lymph node metastases. As shown in Fig. 4D, when PIK3CD mRNA levels were plotted against miR-30a expression, a significant inverse correlation was observed $(\mathrm{r}=-0.782 ; \mathrm{p}<0.001)$. 


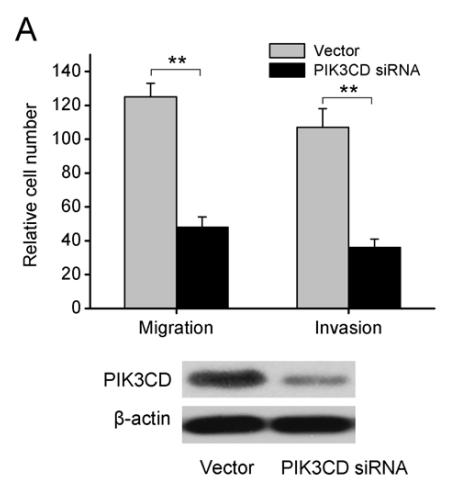

C

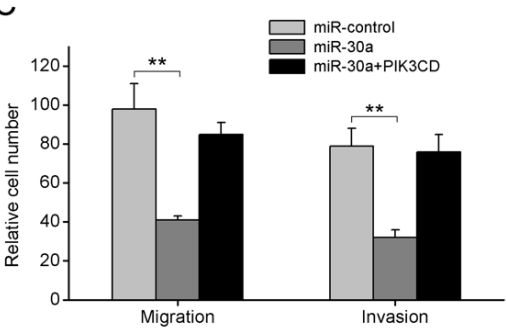

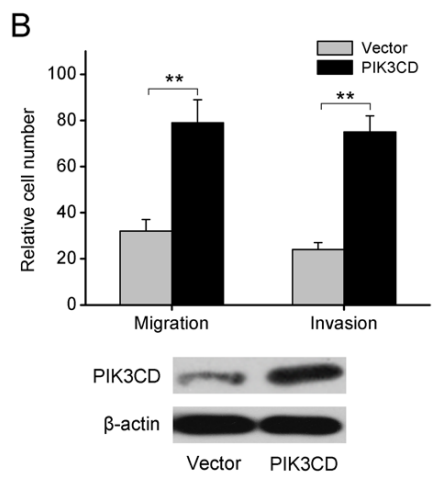

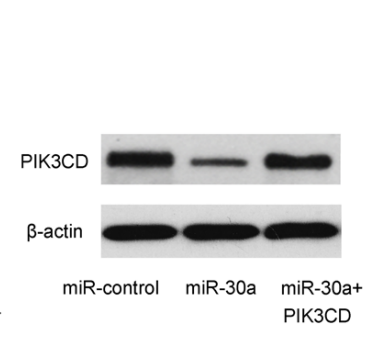

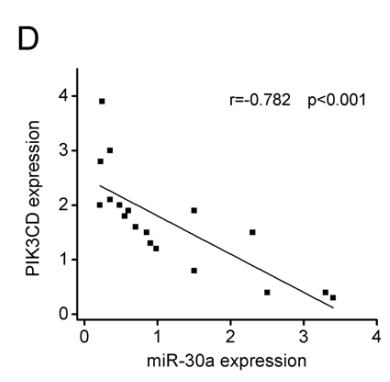

E

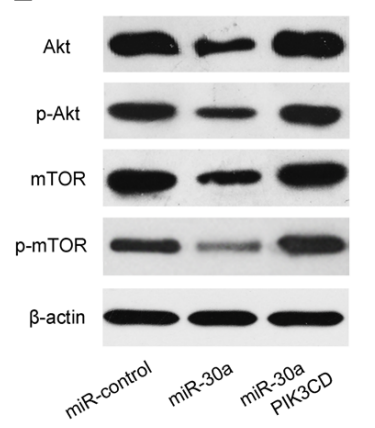

Fig. 4. miR-30a suppresses CRC cell migration and invasion by directly targeting PIK3CD and subsequently affecting PI3K/Akt/mTOR pathway. (A) SW620 cells were infected with PIK3CD siRNA or negative control. Cell migration and invasion were measured by Transwell assays. (B) SW480 cells were transfected with PIK3CD plasmid or vector control, and Transwell migration and invasion assays were then performed. (C) SW620 cells were infected with miR-30a or miR-control, followed by transfection with PIK3CD plasmid, and Transwell migration and invasion assays were then performed. (D) Correlation of miR-30a expression and PIK3CD expression in 18 CRC samples with lymph node metastases. The expression of miR-30a was examined using qRT-PCR analysis and normalized by U6 expression. The expression of PIK3CD was quantified by qRT-PCR and normalized by $\beta$-actin expression. (E) Western blotting analysis of the protein levels of Akt and mTOR genes in SW480 cells infected with miR-30a or miR-control or miR-30a infection, in combination with PIK3CD transfection.

\section{miR-30a regulates the Akt/mTOR pathway}

Next, we investigated the effect of miR-30a down-regulation of PIK3CD expression on the Akt/mTOR pathway. Analysis of Akt, phospho-Akt (p-Akt), mTOR, and phospho-mTOR (p-mTOR) by Western blotting showed that the levels of these proteins were significantly decreased in miR-30a-overexpressed cells compared to control cells, and this downregulation was reversed by PIK3CD overexpression (Fig. 4E). These results indicate that miR-30a plays an important role in the regulation of the Akt/mTOR pathway.

\section{Discussion}

Metastasis is responsible for most cancer mortality. Therefore, it is essential to identify metastasis-associated molecules and to better understand the mechanism behind cancer metastasis. Recently, mounting evidence indicates that miRNAs are involved in advanced stages of cancer progression and that they can function as activators or suppressors of metastasis, including miR-31, miR-34a and miR-146a [18-20]. However, the functional role of miRNAs in CRC metastasis is still largely unknown. In this study, we investigated the biological role of miR-30a in human CRC metastasis. We found that miR-30a was downregulated in metastatic CRC cells compared with non-metastatic cells. Furthermore, miR-30a expression 
was lower in invasive CRC patients with lymph node metastases compared with those of lymph-node-negative patients, suggesting that miR-30a might be involved in both oncogenic transformation and tumor metastasis. Indeed, our results confirm that miR-30a could significantly suppress CRC cell invasion and metastasis both in vitro and in vivo.

Generally, miRNAs exert their function by regulating expression of their downstream target gene(s). miR-30a has been reported to negatively regulate Beclin 1 mRNA in tumor cells resulting in decreased autophagic activity [21, 22]. Futhermore, the role of miR-30a in inhibition of cellular invasion was recently reported in various cancers, including nonsmall cell lung cancer, ewing sarcoma and breast cancer $[15,23,14]$. In particular, Baraniskin and colleagues found that miR-30a-5p targets DTL and suppresses tumor growth in colon carcinoma [24]. However, few studies have been performed to determine which genes miR-30a targets to modulate the behavior of CRC metastasis. In this study, we focused on PIK3CD because of its important role as a regulator of cell growth, invasion and migration [25-27]. We found that miR-30a directly bound to the 3'UTR of PIK3CD, which contains a miR-30a-binding site, by dual-luciferase reporter assay. Moreover, miR-30a overexpression significantly down-regulated PIK3CD expression at both mRNA and protein levels. These results suggest that PIK3CD may be a target of miR-30a in CRC cells.

Recently, several miRNAs have been reported to directly target or regulate PIK3CD, including miR-7, miR-384-5p and miR-125b [16, 28, 29]. These studies revealed that multiple miRNAs probably result in the loss of PIK3CD expression in specific types of cancers, and subsequently inhibit the tumorigenesis; however, only miR-7 has been reported to reverse the metastasis of HCC by targeting PIK3CD [16]. In this study, we provide the first evidence that PIK3CD was frequently overexpressed in lymph node metastatic CRC tissues, knockdown of PIK3CD greatly repressed invasion and migration, suggesting a role of PIK3CD in the progression of CRC. Furthermore, we identified PIK3CD as a direct and functional target of miR-30a. miR-30a-induced inhibition of migration and invasion could be rescued by overexpression of PIK3CD. We also showed for the first time that the expression of PIK3CD is inversely correlated with miR-30a levels in metastatic CRC tissues. These results confirm that miR-30a functions as an anti-metastatic miRNA in CRC cells by targeting PIK3CD.

Given that PIK3CD is an important component of the PI3K/Akt pathway, which is frequently activated in human cancers $[16,30,31]$, we therefore investigated the effects of miR-30a on the expression of PI3K/Akt/mTOR pathway molecules. We found that overexpression of miR-30a down-regulated the expression of Akt and mTOR and its phosphorylated forms, whereas PIK3CD overexpression reversed this suppressive effect. These results further verify that the inhibitory properties of miR-30a against CRC metastasis is mediated, at least in part, by the inhibition of PI3K/Akt/mTOR signaling pathway. However, our results failed to illustrate the mechanism of PIK3CD through PI3K/Akt/mTOR pathway to reduce CRC metastasis. It is probable that cell apoptosis plays an important role in this process [16]. Further studies of the mechanisms underlying this process are required.

In summary, our study provided the first evidence of the biological role of miR-30a in human CRC metastasis, and identified PIK3CD as a target potentially involved in miR-30amediated suppression of CRC cell migration and invasion via the PI3K/Akt/mTOR pathway. These findings may provide the basis for the future use of miR-30a in the treatment of CRC.

\section{Disclosure}

All authors are in agreement with the content of this manuscript. The authors declare no conflict of interest. 
Zhong/Bian/Wu: miR-30a Targets PIK3CD Expression in CRC

\section{References}

Jemal A, Siegel R, Xu J, Ward E: Cancer statistics. CA Cancer J Clin 2010;60:277-300.

Ambros V: The functions of animal microRNAs. Nature 2004;431:350-355.

Lu J, Getz G, Miska EA, Alvarez-Saavedra E, Lamb J, Peck D, Sweet-Cordero A, Ebert BL, Mak RH, Ferrando AA, Downing JR, Jacks T, Horvitz HR, Golub TR: MicroRNA expression profiles classify human cancers. Nature 2005; 435:834-838.

4 Calin GA, Croce CM: MicroRNA signatures in human cancers. Nat Rev Cancer 2006;6:857-866.

-5 White NM, Fatoohi E, Metias M, Jung K, Stephan C, Yousef GM: Metastamirs: a stepping stone towards improved cancer management. Nat Rev Clin Oncol 2011;8:75-84.

6 Osaki M, Takeshita F, Sugimoto Y, Kosaka N, Yamamoto Y, Yoshioka Y, Kobayashi E, Yamada T, Kawai A, Inoue T, Ito H, Oshimura M, Ochiya T: MicroRNA-143 regulates human osteosarcoma metastasis by regulating matrix metalloprotease-13 expression. Mol Ther 2011;19:1123-1130.

7 Zhang Y, Wang Z, Chen M, Peng L, Wang X, Ma Q, Ma F, Jiang B: MicroRNA-143 targets MACC1 to inhibit cell invasion and migration in colorectal cancer. Mol Cancer 2012;11:23.

8 Hu Y, Ou Y, Wu K, Chen Y, Sun W: miR-143 inhibits the metastasis of pancreatic cancer and an associated signaling pathway. Tumour Biol 2012;33:1863-1870.

-9 Shen K, Liang Q, Xu K, Cui D, Jiang L, Yin P, Lu Y, Li Q, Liu J: MiR-139 inhibits invasion and metastasis of colorectal cancer by targeting the type I insulin-like growth factor receptor. Biochem Pharmacol 2012;84:320-330.

10 Xu X, Chen Z, Zhao X, Wang J, Ding D, Wang Z, Tan F, Tan X, Zhou F, Sun J, Sun N, Gao Y, Shao K, Li N, Qiu B, He J: MicroRNA-25 promotes cell migration and invasion in esophageal squamous cell carcinoma. Biochem Biophys Res Commun 2012;421:640-645.

-11 Cummins JM, He Y, Leary RJ, Pagliarini R, Diaz LA Jr, Sjoblom T, Barad O, Bentwich Z, Szafranska AE, Labourier E, Raymond CK, Roberts BS, Juhl H, Kinzler KW, Vogelstein B, Velculescu VE: The colorectal microRNAome. Proc Natl Acad Sci USA 2006;103:3687-3692.

12 Sarver AL, French AJ, Borralho PM, Thayanithy V, Oberg AL, Silverstein KA, Morlan BW, Riska SM, Boardman LA, Cunningham JM, Subramanian S, Wang L, Smyrk TC, Rodrigues CM, Thibodeau SN, Steer CJ: Human colon cancer profiles show differential microRNA expression depending on mismatch repair status and are characteristic of undifferentiated proliferative states. BMC Cancer 2009;9:401.

13 Arndt GM, Dossey L, Cullen LM, Lai A, Druker R, Eisbacher M, Zhang C, Tran N, Fan H, Retzlaff K, Bittner A, Raponi M: Characterization of global microRNA expression reveals oncogenic potential of miR-145 in metastatic colorectal cancer. BMC Cancer 2009;9:374.

14 Cheng CW, Wang HW, Chang CW, Chu HW, Chen CY, Yu JC, Chao JI, Liu HF, Ding SL, Shen CY: MicroRNA-30a inhibits cell migration and invasion by downregulating vimentin expression and is a potential prognostic marker in breast cancer. Breast Cancer Res Treat 2012;134:1081-1093.

15 Kumarswamy R, Mudduluru G, Ceppi P, Muppala S, Kozlowski M, Niklinski J, Papotti M, Allgayer H: MicroRNA-30a inhibits epithelial-to-mesenchymal transition by targeting Snai1 and is downregulated in non-small cell lung cancer. Int J Cancer 2012;130:2044-2053.

16 Fang Y, Xue JL, Shen Q, Chen J, Tian L: MicroRNA-7 inhibits tumor growth and metastasis by targeting the phosphoinositide 3-kinase/Akt pathway in hepatocellular carcinoma. Hepatology 2012;55:1852-1862.

17 Lv XB, Jiao Y, Qing Y, Hu H, Cui X, Lin T, Song E, Yu F: miR-124 suppresses multiple steps of breast cancer metastasis by targeting a cohort of pro-metastatic genes in vitro. Chin J Cancer 2011;30:821-830.

18 Valastyan S, Reinhardt F, Benaich N, Calogrias D, Szász AM, Wang ZC, Brock JE, Richardson AL, Weinberg RA: A pleiotropically acting microRNA, miR-31, inhibits breast cancer metastasis. Cell 2009;137:10321046.

19 Liu C, Kelnar K, Liu B, Chen X, Calhoun-Davis T, Li H, Patrawala L, Yan H, Jeter C, Honorio S, Wiggins JF, Bader AG, Fagin R, Brown D, Tang DG: The microRNA miR-34a inhibits prostate cancer stem cells and metastasis by directly repressing CD44. Nat Med 2011;17:211-215.

20 Li Y, Vandenboom TG 2nd, Wang Z, Kong D, Ali S, Philip PA, Sarkar FH: miR-146a suppresses invasion of pancreatic cancer cells. Cancer Res 2010;70:1486-1495.

21 Zhu H, Wu H, Liu X, Li B, Chen Y, Ren X, Liu CG, Yang JM: Regulation of autophagy by a beclin 1-targeted microRNA, miR-30a, in cancer cells. Autophagy 2009;5:816-823. 
22 Zou Z, Wu L, Ding H, Wang Y, Zhang Y, Chen X, Chen X, Zhang CY, Zhang Q, Zen K: MicroRNA-30a sensitizes tumor cells to cis-platinum via suppressing beclin 1-mediated autophagy. J Biol Chem 2012;287:41484156.

23 Franzetti GA, Laud-Duval K, Bellanger D, Stern MH, Sastre-Garau X, Delattre O: MiR-30a-5p connects EWS-FLI1 and CD99, two major therapeutic targets in Ewing tumor. Oncogene (2012) doi: 10.1038/ onc.2012.403.

-24 Baraniskin A, Birkenkamp-Demtroder K, Maghnouj A, Zöllner H, Munding J, Klein-Scory S, ReinacherSchick A, Schwarte-Waldhoff I, Schmiegel W, Hahn SA: MiR-30a-5p suppresses tumor growth in colon carcinoma by targeting DTL. Carcinogenesis 2012;33:732-739.

25 Fransson S, Uv A, Eriksson H, Andersson MK, Wettergren Y, Bergo M, Ejeskär K: p37 $\delta$ is a new isoform of $\mathrm{PI} 3 \mathrm{~K}$ p110 $\delta$ that increases cell proliferation and is overexpressed in tumors. Oncogene 2012;31:32773286.

-26 Foukas LC, Berenjeno IM, Gray A, Khwaja A, Vanhaesebroeck B: Activity of any class IA PI3K isoform can sustain cell proliferation and survival. Proc Natl Acad Sci USA 2010;107:11381-11386.

27 Billottet C, Grandage VL, Gale RE, Quattropani A, Rommel C, Vanhaesebroeck B, Khwaja A: A selective inhibitor of the p110delta isoform of PI3-kinase inhibits AML cell proliferation and survival and increases the cytotoxic effects of VP16. Oncogene 2006;25:6648-6659.

28 Bao Y, Lin C, Ren J, Liu J: MicroRNA-384-5p regulates ischemia-induced cardioprotection by targeting phosphatidylinositol-4,5-bisphosphate 3-kinase, catalytic subunit delta (PI3K p1108). Apoptosis 2013; in press.

29 Cui F, Li X, Zhu X, Huang L, Huang Y, Mao C, Yan Q Zhu J, Zhao W, Shi H: MiR-125b inhibits tumor growth and promotes apoptosis of cervical cancer cells by targeting phosphoinositide 3-kinase catalytic subunit delta. Cell Physiol Biochem 2012;30:1310-1318.

30 Boller D, Schramm A, Doepfner KT, Shalaby T, von Bueren AO, Eggert A, Grotzer MA, Arcaro A: Targeting the phosphoinositide 3-kinase isoform p110 $\mathrm{impairs}$ growth and survival in neuroblastoma cells. Clin Cancer Res 2008;14:1172-1181.

31 Park S, Chapuis N, Tamburini J, Bardet V, Cornillet-Lefebvre P, Willems L, Green A, Mayeux P, Lacombe C, Bouscary D: Role of the PI3K/AKT and mTOR signaling pathways in acute myeloid leukemia. Haematologica 2010;95:819-828. 